\title{
Emergence of Lamina-Specific Retinal Ganglion Cell Connectivity by Axon Arbor Retraction and Synapse Elimination
}

\author{
Ting-Wen Cheng, ${ }^{1 \star}$ Xiao-Bo Liu, ${ }^{1 \star}$ Regina L. Faulkner, ${ }^{1}$ Alexander H. Stephan, ${ }^{2}$ Ben A. Barres, ${ }^{2}$ \\ Andrew D. Huberman, ${ }^{3,4}$ and Hwai-Jong Cheng ${ }^{1}$ \\ ${ }^{1}$ Center for Neuroscience, University of California, Davis, California 95618, ${ }^{2}$ Department of Neurobiology, Stanford University School of Medicine, Palo \\ Alto, California 94305, and ${ }^{3}$ Neurosciences Department, School of Medicine, and ${ }^{4}$ Neurobiology Section, Division of Biological Sciences, University of \\ California, San Diego, La Jolla, California 92093
}

Throughout the nervous system, neurons restrict their connections to specific depths or "layers" of their targets to constrain the type and number of synapses they make. Despite the importance of lamina-specific synaptic connectivity, the mechanisms that give rise to this feature in mammals remain poorly understood. Here we examined the cellular events underlying the formation of lamina-specific retinal ganglion cell (RGC) axonal projections to the superior colliculus (SC) of the mouse. By combining a genetically encoded marker of a defined RGC subtype (OFF- $\alpha$ RGCs) with serial immunoelectron microscopy, we resolved the ultrastructure of axon terminals fated for laminar stabilization versus those fated for removal. We found that OFF- $\alpha$ RGCs form synapses across the full depth of the retinorecipient SC before undergoing lamina-specific arbor retraction and synapse elimination to arrive at their mature, restricted pattern of connectivity. Interestingly, we did not observe evidence of axon degeneration or glia-induced synapse engulfment during this process. These findings indicate that lamina-specific visual connections are generated through the selective stabilization of correctly targeted axon arbors and suggest that the decision to maintain or eliminate an axonal projection reflects the molecular compatibility of presynaptic and postsynaptic neurons at a given laminar depth.

\section{Introduction}

Precise synaptic connections are essential for neural circuit function. How do developing neurons distinguish among potential synaptic partners and make connections with appropriate target cells? Long-range and topographic guidance cues restrict the general number and type of synaptic partners (Tessier-Lavigne and Goodman, 1996; McLaughlin and O'Leary, 2005), but mechanisms that operate over a finer spatial scale ensure synaptic specificity. One such mechanism is laminar specificity; throughout the CNS, neurons are organized into parallel layers that contain distinct types or portions of neurons. By directing axonal and dendritic processes to specific layers, a high degree of synaptic specificity is achieved (Sanes and Yamagata, 2009; Huberman et al., 2010).

Lamina-specific axon targeting has been studied extensively in Drosophila visual system (Clandinin and Zipursky, 2002), but in mammals, little is known about the mechanisms that direct axons from different functional classes of neurons into distinct laminae.

Received July 3, 2010; revised 0ct. 1, 2010; accepted Oct. 7, 2010.

This work was supported by National Institute of Child Health and Human Development Grant HD045757 and the March of Dimes Foundation (H.-J.C.), National Eye Institute Grants R21 EY018320 and EY11310 (B.A.B.), National Institute on Drug Abuse Grant DA15043 (B.A.B), and a grant from Wyeth Pharmaceuticals (B.A.B). A. Stephan was supported by a Swiss National Science Foundation fellowship. We thank Edward G. Jones for support and comments. *T.-W.C. and X.-B.L. contributed equally to this work.

Correspondence should be addressed to Hwai-Jong Cheng at the above address. E-mail: hjcheng@ucdavis.edu. DOI:10.1523/JNEUROSCI.3455-10.2010

Copyright $\odot 2010$ the authors $\quad 0270-6474 / 10 / 3016376-07 \$ 15.00 / 0$
A study in hamsters concluded that the axons of the output neurons of the eye, retinal ganglion cells (RGCs), first project broadly across the depth of retinorecipient superior colliculus (SC) before they refine to the correct layer (Sachs et al., 1986). By contrast, a study of fetal macaque monkeys showed that laminaspecific RGC projections are generated through accurate targeting, not pruning (Meissirel et al., 1997). The interpretations of both those studies were complicated, however, by a lack of techniques that could identify specific RGC axons as destined to connect to particular target layers.

The recent discovery of genetic markers for specific RGC subtypes (Huberman et al., 2008a, 2009; Siegert et al., 2009; Yonehara et al., 2009; Kim et al., 2010) has created the opportunity to directly probe the mechanisms that generate lamina-specific synaptic connections. Previously we found that OFF- $\alpha$ RGC axons trespass into inappropriate laminae of the SC before refining to the correct laminar depth (Huberman et al., 2008a). A subsequent study confirmed this finding for other RGC subtypes and showed that some axons target their correct layer from the outset (Kim et al., 2010), as they do in zebrafish (Nevin et al., 2008). However, several important questions about the cellular mechanisms underlying lamina-specific RGC axon targeting remain unanswered. For instance, does the generation of lamina-specific connectivity reflect synapse formation and elimination-as is the case for eye-specific targeting (Campbell and Shatz, 1992; Jaubert-Miazza et al., 2005; Bickford et al., 2010)—and if so, are the synapses that are fated for removal structurally different from 
the synapses that are fated for stabilization? Given the longstanding prominence of RGC axon projections as a model for understanding CNS circuit development, it is crucial that the cellular mechanisms that generate lamina-specific targeting are resolved.

Here we performed serial immuno-electron microscopy (immuno-EM) on the SC of mice in which a defined RGC subtype selectively and stably expresses green fluorescent protein (GFP). We find that lamina-specific retino-SC connections emerge through a balance of synapse elimination, axon arbor retraction, and terminal stabilization. We discuss our findings in terms of their potential relevance to underlying activity dependence and molecular mechanisms.

\section{Materials and Methods}

Animals, tissue preparation, and quantification of immunofluorescent microscopy. Calbindin2-EGFP (CB2-GFP) mice were obtained from the Mutant MouseRegionalResourceCenters(http://www.mmrrc.org/strains/283/0283. $\mathrm{html}$ ) and in some cases mated with complement-deficient (Clq knock-out) mice (Botto et al., 1998) to generate CB2-GFP::C1q ${ }^{-1-}$ mice. Mice were perfused and postfixed overnight in $4 \%$ paraformaldehyde before immunohistochemistry processing as by Huberman et al. (2008a) for anti-GFP (1: 2000; Invitrogen) and anti-Ibal (1:500; Wako). Images were taken with Zeiss M1 Axioscope (Fig. 1) or LSM 510 confocal microscope (Fig. 3A-I). For quantification of Ibal + glia, three animals at each time point after enucleation were analyzed. The mean number of cells was measured from three coronal sections across the anterior-posterior axis of retinorecipient SC. All procedures in this report were in accordance with animal care and use protocols at Stanford University School of Medicine and UC Davis.

Monocular enucleation to induce RGC axon degeneration. Postnatal day 8 (P8) CB2-GFP mice $(n=11)$ were anesthetized with inhalant isoflurane, the eyelids were gently parted with blunt tweezers, and the extraocular muscles and optic nerve were cut with fine iridectomy scissors. Gelfoam was applied to the enucleated cavity to prevent bleeding and the eyelid sealed with Vetbond. The pup was returned to its mother litter and monitored every 30 min over the subsequent $4-6 \mathrm{~h}$, then overdosed with sodium pentobarbital, and perfused for light and electron microscopy.

Immuno-EM. CB2-GFP mice aged P4, P6, P8, or P11 ( $n=3-4$ mice per age) were used for EM. Visualization of GFP+ axons by EM was performed as described by Faulkner et al. (2008). Briefly, mice were perfused with saline followed by $4 \%$ paraformaldehyde plus $0.1 \%$ glutaraldehyde, then postfixed overnight in $4 \%$ paraformaldehyde. Brain sections ( $\sim 50 \mu \mathrm{m}$ thick) were processed with anti-GFP (1:2000, Invitrogen) using $0.02 \%$ Triton X-100 to facilitate antibody penetration $(\sim 9 \mu \mathrm{m}$ on either surface) and visualized with DAB histochemistry. Dehydrated and flat-embedded tissues were processed for serial ultrathin sectioning $(\sim 70$ $\mathrm{nm}$ ) (Leica Ultracut) and examined with a Philips CM120 electron microscope. Images were acquired with a $2000 \times 2000$ pixel CCD camera (Gatan).

EM quantification and statistical analysis. Bouton reconstructions were made from serial ultrathin sections $(2-8$ sections per bouton; mean $=$ 3.8 serial sections). Bouton perimeters were measured from the ultrathin section in which the bouton was largest (using NIH ImageJ). The bouton's laminar position was determined by measuring its distance from the SC pial surface using photo-montages of low-magnification photomicrographs. GFP boutons located in the superficial-most $150 \mu \mathrm{m}$ of the stratum griseum superficialis (SGS) of the SC were defined as upper SGS (uSGS) boutons, whereas GFP boutons located $>150 \mu \mathrm{m}$ from the pia were defined as in the lower SGS (ISGS). A synapse (presynaptic vesicles opposed by a synaptic cleft and a postsynaptic density) was identified when the synaptic profile was evident in two or more serial sections of a reconstructed bouton. PSD length was measured from the ultrathin section in which the PSD was longest. When more than one synapse was associated with a bouton, average PSD length of all synapses was reported. For lesion-induced degeneration experiments, GFP fragments were delineated on the basis of electron-dense products that were aligned across multiple serial sections. The border between the electron-lucent area encompassing the GFP fragments and the surrounding neuropil was used to delineate the glial cell processes. For 3D reconstructions, serial micrographs were traced and reconstructed with Reconstruct (Synapse Web). 3ds Max (Autodesk) was used for rendering. Statistical analyses were calculated with Microsoft Excel and IBM SPSS Statistics. Multiple comparisons were analyzed with ANOVA statistics and Hochberg post hoc comparison tests.

\section{Results}

\section{Stereotyped lamina-specific axonal refinement in the SC}

To study the cellular mechanisms that underlie lamina-specific axonal refinement, we used CB2-GFP transgenic mice. In this mouse line, GFP is selectively expressed by OFF- $\alpha$ RGCs. This allows visualization of OFF- $\alpha$ RGC axons in retinorecipient targets across development and into adulthood (Huberman et al., 2008a). Previous studies have shown that from P0 until P8, RGC axons undergo retinotopic refinement, with the major phase of anatomical remodeling occurring between P0 and P4 (McLaughlin and O'Leary, 2005). Also from P0 to P4, SC neurons complete their migration into the appropriate laminae (Edwards et al., 1986) (Fig. 1A). Subsequently, lamina-specific refinement ensues: from $\mathrm{P} 4$ to $\mathrm{P} 6$, OFF- $\alpha$ RGCs project axons broadly across the depth of retinorecipient SC and then from P7 to P12, OFF- $\alpha$ RGC axons are removed from the uSGS. Thus by $\mathrm{P} 12$, virtually all OFF- $\alpha$ RGC axons are found in the ISGS (Fig. 1A-E) (Huberman et al., 2008a). The stereotyped refinement of axons arising from a RGC population expressing a stable genetic marker provides a unique opportunity to examine the ultrastructural changes associated with lamina-specific axon targeting and to directly compare the ultrastructure of RGC axon projections destined for removal versus those destined for laminar stabilization.

\section{Ultrastructure of lamina-specific projections to the SC during refinement}

To explore the ultrastructural changes that underlie laminaspecific axonal refinement, we analyzed GFP-expressing (GFP+) OFF- $\alpha$ RGC axons in the SC of developing mice, using serialsection immuno-EM. First we focused on axonal boutons, because they are a well defined structural correlate of presynaptic terminals. GFP + axons in the superficial-most $150 \mu \mathrm{m}$ of the SC are defined as axons in the uSGS that are fated for removal, because virtually all such profiles are pruned from this lamina by P11/12 (Fig. 1) (Huberman et al., 2008a). EM analysis revealed that GFP + boutons in the uSGS increased in average size by $83 \%$ between $\mathrm{P} 4$ and $\mathrm{P} 6$, followed by a continuous decrease in average size $(\sim 41 \%)$ from P6 to P11 (Fig. $2 A, B)(\mathrm{P} 4 / \mathrm{P} 6 p=0.003, \mathrm{P} 6 /$ $\mathrm{P} 11 p=0.007$, one-way ANOVA Hochberg tests; $n=12-17)$. By contrast, the average size of GFP+ boutons in the ISGS (those fated for stabilization) remained stable throughout the major phase of lamina-specific targeting (Fig. $2 A, B)(p=0.20$, oneway ANOVA; $n=12-18)$. Interestingly, throughout the period of laminar refinement (P4-P11), GFP+ boutons in the USGS were significantly smaller than the GFP + boutons targeted to the ISGS (Fig. $2 B)(p=0.01$, two-way ANOVA; $n=56-62)$. This could arise because the more distal portions of the GFP + axons are thinner, due to molecular differences in boutons fated for removal versus stabilization (see Discussion), or both. Regardless, our data indicate that axon boutons that are transiently targeted to inappropriate layers fail to achieve the full size of axon boutons that are targeted to their correct lamina. 
A

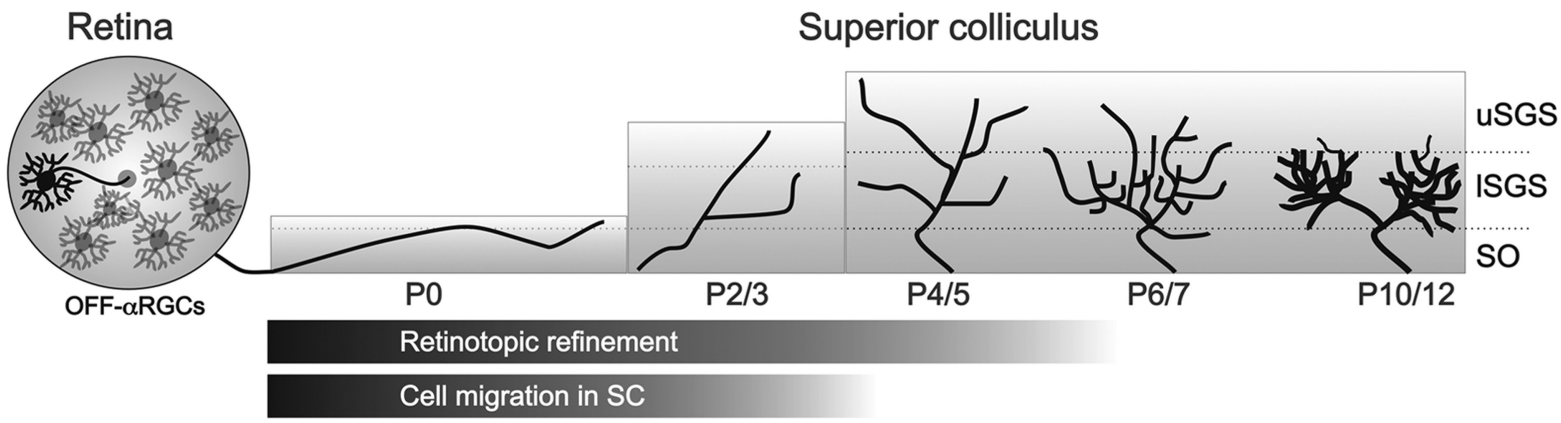

Lamina-specific refinement

B

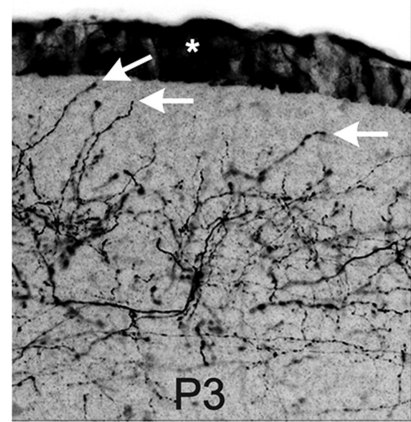

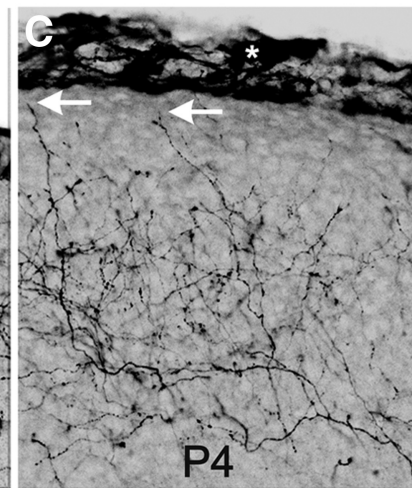

D

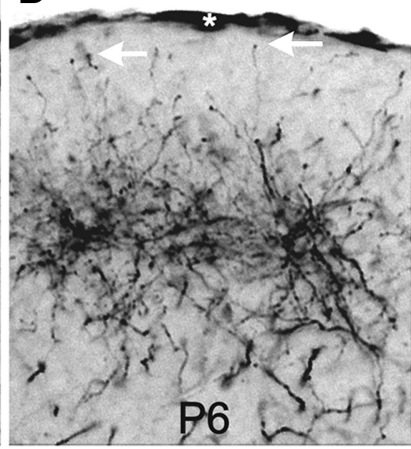

$E$

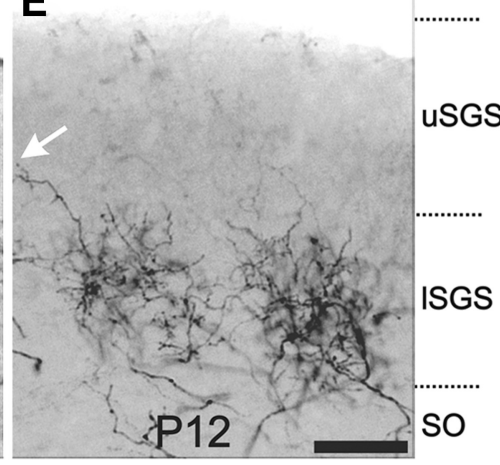

Figure 1. Lamina-specific axonal refinement of genetically identified OFF- $\alpha \mathrm{RGCS}$. $A$, Schematic of retinotopic refinement, SC lamina development, and lamina-specific axon targeting for OFF- $\alpha$ RGCS in the mouse. Retinotopy and SC laminae develop by P4/5, whereas lamina-specific refinement occurs from P4/5 to P12 (Edwards et al., 1986; McLaughlin and 0'Leary, 2005; Huberman et al., 2008a). $\boldsymbol{B}-\boldsymbol{E}$, Lamina-specific refinement of GFP + OFF- $\alpha$ RGC axons in the SC. Arrows, GFP + axons in the uSGS that are destined for removal. Asterisk, Non-neural cells that transiently express GFP at the pia. S0, Stratum opticum. Scale bar (in $\boldsymbol{E}$ ): $\boldsymbol{B}-\boldsymbol{D}, 100 \mu \mathrm{m} ; \boldsymbol{E}, 150 \mu \mathrm{m}$.

\section{Structural synapse formation and elimination underlie lamina-specific targeting}

Next we asked whether lamina-specific refinement involves synapse formation and elimination. We defined a synapse as a GFP-labeled profile with the following: (1) a GFP+ boutoncontaining synaptic vesicles, (2) an opposing synaptic cleft, and (3) a postsynaptic density. Using those criteria, we first observed $\mathrm{GFP}+$ synapses in the retinorecipient SC at P6. At this age, synapses were present in both uSGS and ISGS (Fig. 2A). Indeed, the percentage of GFP + boutons making synapses was very similar in the uSGS versus ISGS at P6 (Fig. 2C) [uSGS, 47\% $(n=7 / 15)$; ISGS, $50 \%(n=8 / 16)]$. From P8 to P11, most GFP+ axons were removed from the uSGS, but a minority persisted there (Fig. $1 E$ ). Interestingly, the GFP + axons that persisted in the uSGS contained synapses at percentages only slightly lower than GFP+ inputs to the ISGS at the same age [P8: uSGS $47 \%(n=8 / 17)$, ISGS 56\% $(n=9 / 16)$; P11: uSGS $42 \%(n=5 / 12)$, ISGS 50\% $(n=$ $9 / 18)$ ]. Overall, these data indicate that early in development, OFF- $\alpha$ RGC axons form synaptic contacts with cells in both correct and incorrect target laminae. Given that virtually all GFP+ axonal inputs to the uSGS are removed by P12 (Fig. 1), laminaspecific refinement of OFF- $\alpha$ RGC axons must involve synapse elimination.

\section{Axonal degeneration during lesion-induced but not developmental lamina-specific pruning}

How are OFF- $\alpha$ RGC axons and synapses removed from the uSGS? Neither our fluorescent microscopic nor EM analysis revealed any immediate signs of axonal degeneration or glial engulfment during laminar retino-SC refinement (Figs. 1, 2). In $\mathrm{CB} 2$ mice, GFP + OFF- $\alpha$ RGCs in each eye project only to the contralateral SC, so to more closely evaluate whether laminaspecific refinement involves axon degeneration, we removed one eye from P8 CB2-GFP mice and compared the GFP+ axons in the SC ipsilateral and contralateral to the enucleated eye. Four hours after enucleation, fragmented GFP + axons were readily observed in the contralateral (deafferented) SC but not in the ipsilateral (control) SC (Fig. $3 A, B$ ). The axon fragmentation was also accompanied by a significant increase in Ibal + microglia $4-6 \mathrm{~h}$ after enucleation (Fig. $3 I, J)(p=0.03$, two-way ANOVA; $n=3$ for each time point). Moreover, in CB2-GFP mice that also lacked the immune protein $\mathrm{C} 1 \mathrm{q}\left(\mathrm{CB} 2-\mathrm{GFP}:: \mathrm{Clq}^{-/-}\right)$, laminaspecific pruning proceeded normally (Fig. $3 K, L$ ). C1q is important for microglia-mediated synapse elimination during eye-specific refinement (Stevens et al., 2007). Thus, the lack of impairment in lamina-specific pruning in CB2-GFP::C1q ${ }^{-1-}$ mice further supports the idea that this process is gliaindependent. Indeed, when we compared the ultrastructure of GFP+ boutons in the uSGS $5 \mathrm{~h}$ after enucleation, intact boutons with synapses were observed in the ipsilateral (non-deafferented) hemisphere of the SC (Fig. 3M-O). By contrast, contralateral to the enucleated eye, degenerating GFP + fragments surrounded by electron-lucent glial cell processes were readily apparent (Fig. $3 P-R$; and see supplemental movie, available at www.jneurosci. org as supplemental material). Collectively, these data indicate that during normal development, laminar specificity is achieved independently of axonal degeneration or glial engulfment.

Decoupling synaptic maintenance and axonal refinement Our findings that synapse formation and elimination are concurrent with the refinement of OFF- $\alpha$ RGC axons (Figs. 1-3) led us to ask whether the formation and/or maintenance of synaptic 
A
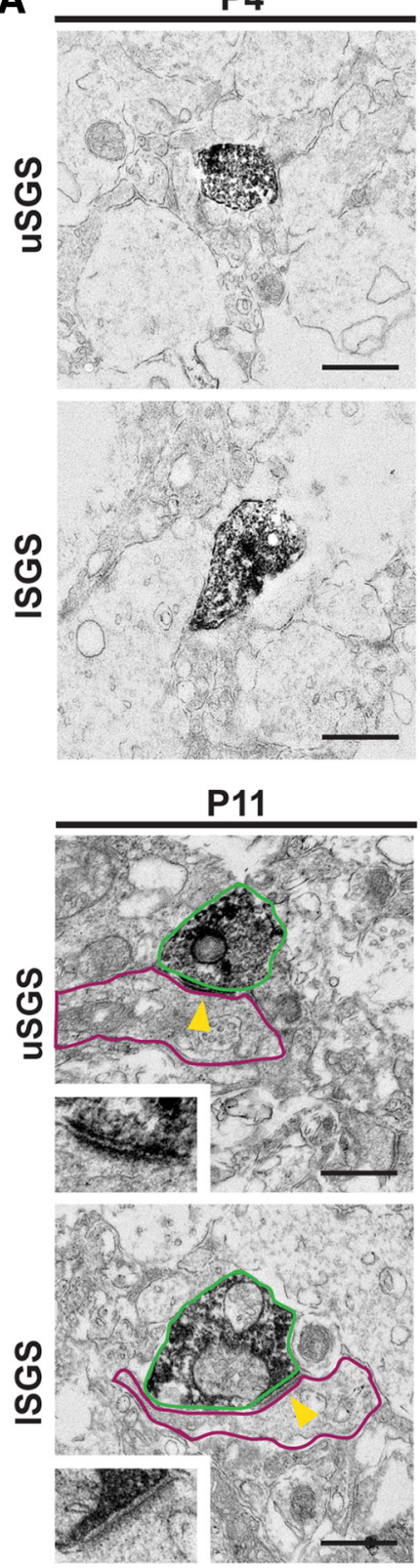

P4

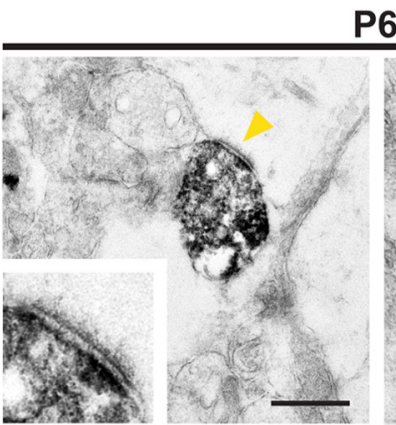

P6
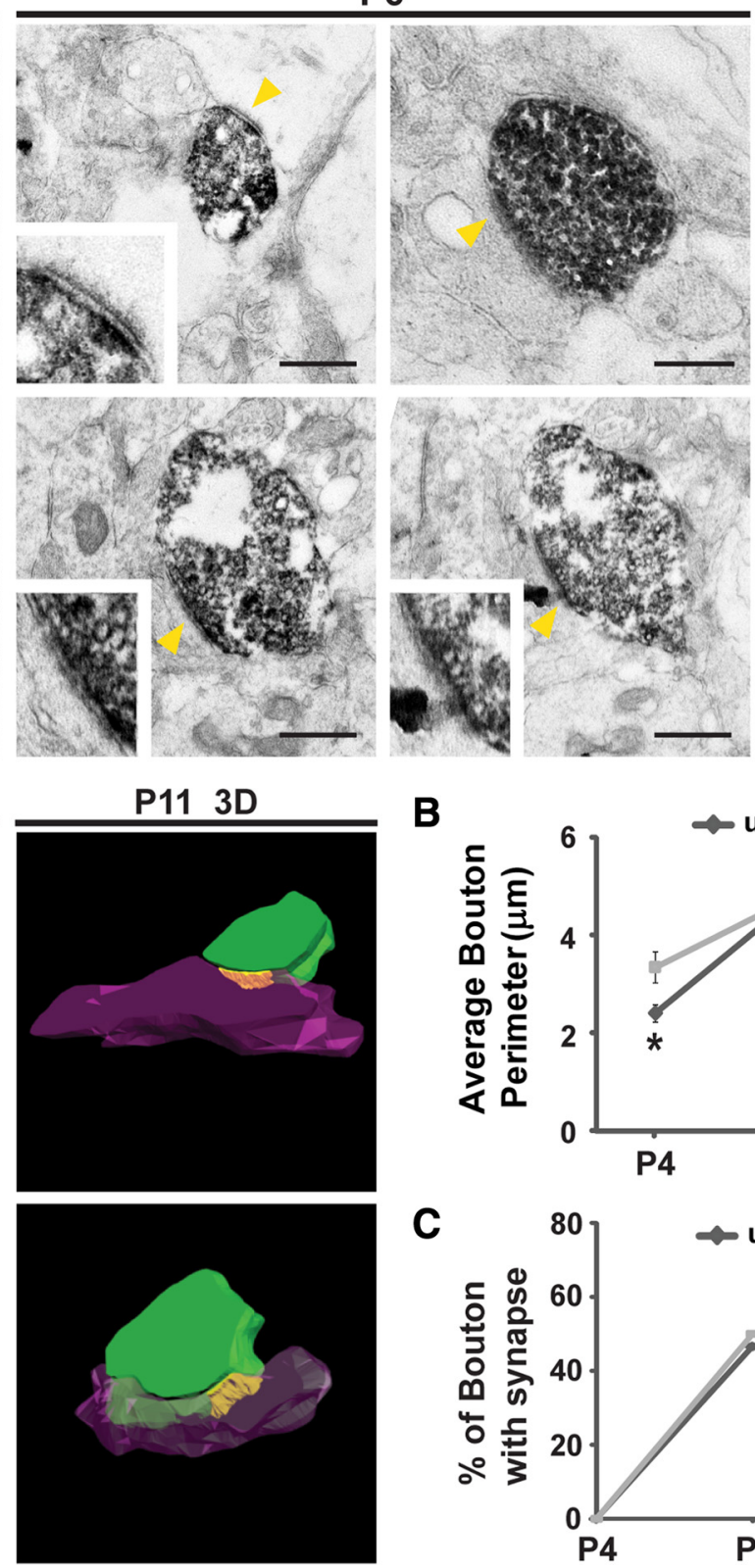

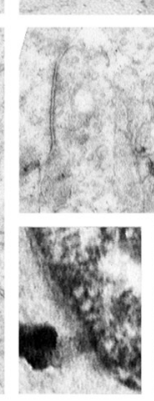

B

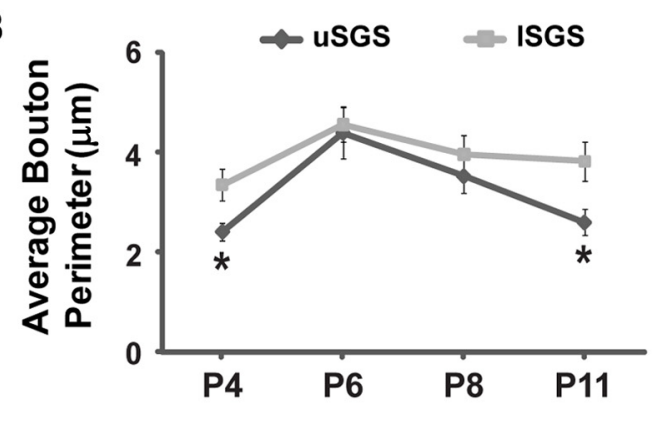

C

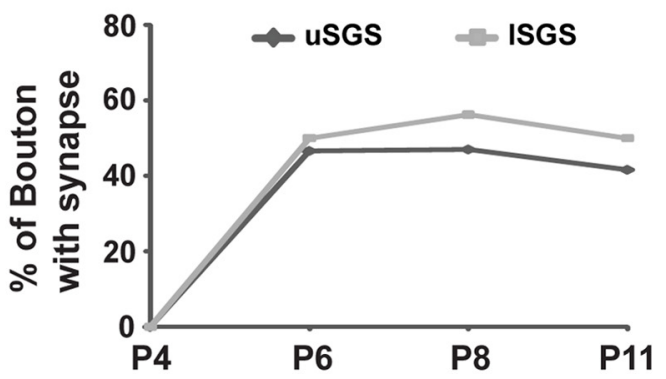

Figure 2. Ultrastructure of boutons formed by developing OFF- $\alpha \mathrm{RGC}$ axons in the SC. $A$, Electron micrographs of GFP + boutons (electron-dense immunoperoxidase reaction products) in the developing SC. GFP + boutons in the uSGS or ISGS of the SC were examined at P4, P6, P8, and P11 (scale bars, $0.5 \mu \mathrm{m}$ ). Insets, High-magnification images of synapses [arrowheads; scale bars (in main panels), $0.2 \mu \mathrm{m}$ ]. Two serial ultrathin sections of the same bouton were presented for P6 ISGS. P11 3D, Serial EM reconstructions of P11 boutons (5- 6 serial ultrathin sections per bouton, 0.35-0.42 $\mu \mathrm{m}$ in thickness) showing the synaptic organization (yellow, PSD) of GFP + boutons (green) and postsynaptic profiles (purple). $\boldsymbol{B}$, GFP + bouton perimeter (mean \pm SEM) as a function of age and laminar position. Boutons in the uSGS are significantly smaller than boutons in the ISGS during laminar refinement ( $p=0.01$, two-way ANOVA; $n=56-62$ ). In the uSGS, bouton size increased from P4 to P6, followed by a reduction from P6 to P11 ( ${ }^{*} p<0.007$, one-way ANOVA Hochberg tests; $n=12-17$ ). C, Percentage of synapses containing boutons from P4 through P11. Synapses were first observed at P6, and similar percentages were detected in both uSGS and ISGS from P4 to P11 ( $n=12-18$ ).

contacts is related to the stability of RGC axonal arbors. We reasoned that if synapse formation is a prerequisite for stabilizing a given arbor, then synaptic maturation might progress faster for boutons in the ISGS than for those in the uSGS. Interestingly, in both the uSGS and ISGS, when the synaptic profiles were present, on average 1 to 2 synaptic contacts were associated per bouton (uSGS: P6 1.00 \pm 0.00, P8 1.38 \pm 0.18, P11 $1.40 \pm 0.16$; 1SGS: P6 $1.25 \pm 0.12$, P8 $1.11 \pm 0.08, \mathrm{P} 111.22 \pm 0.10 ; n=5-9)$. Moreover, PSD lengths were comparable for GFP+ synapses regardless of whether they resided in the uSGS or ISGS (Fig. 4A) ( $p=0.33$, two-way ANOVA; $n=5-9$ ). Indeed, there was no significant increase in synaptic number or size for deep layer boutons during the major phase of arbor stabilization (synaptic number: $p=0.76$, one-way ANOVA; synaptic size: $p=0.12$, one-way ANOVA; $n=8-9)$. These results indicate that the structural maturation of synapses fated for removal highly resembles the maturation of synapses fated for stabilization.

Our observation that bouton size reduced from P6 to P11 (Fig. $2 B$ ) supports the notion that axon retraction is the cellular mechanism for lamina-specific refinement. If the balance of axon growth and retraction is determined primarily by the presence of synapse on a given arbor, then the absence of a synapse might correlate with, and perhaps even predict, a reduction in bouton size. Although we did not analyze individual boutons repeatedly 

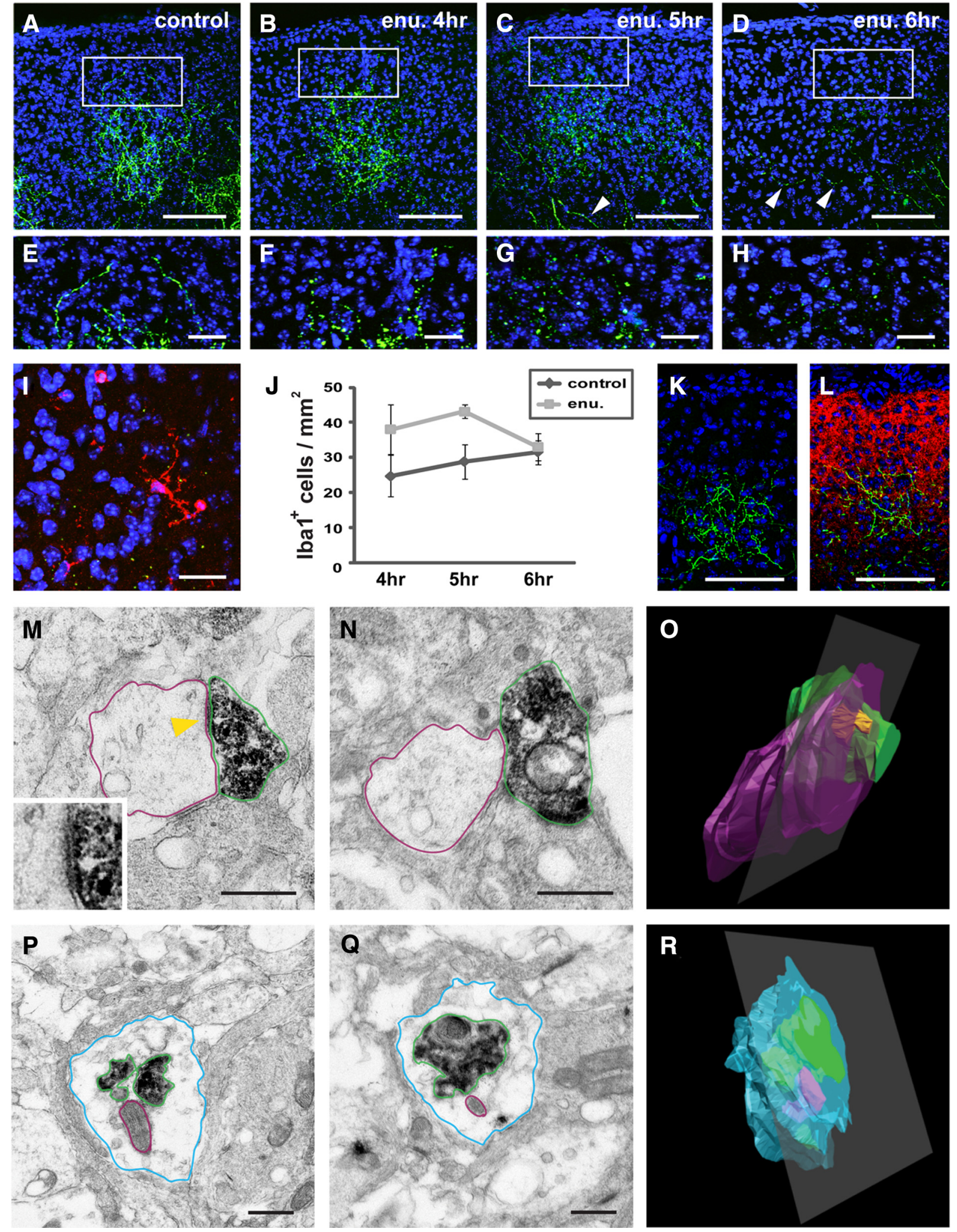

Figure 3. Characteristics of developmental versus lesion-induced RGC axon pruning. $A-H$, Confocal images of the SC of CB2-GFP mice, after unilateral enucleation at P8. GFP + RGC axons (green) and DAPI+ cell bodies (blue), in the SC ipsilateral (control) and contralateral (enu.) to the enucleated eye. High-magnification images of the uSGS (outlined boxes in $\boldsymbol{A}-\boldsymbol{D}$ ) are shown in $\boldsymbol{E}-\boldsymbol{H}$, respectively. Fragmented axons were observed in the enu. SC 4-6 h after enucleation. Degeneration of RGC axons progressed from the distal toward the proximal (arrowheads) aspect of GFP+ axons. II Iba1+ (red) microglia. J, Number of Iba1+ glial cells (mean \pm SEM). More Iba1 + cells were present in the enu. SC versus the control SC $4-6 \mathrm{~h}$ after enucleation ( $p=0.03$, two-way ANOVA; $n=3$ per time point). $K, L, G F P+$ axons in the SC of two P11 C1q- / - ::CB2-GFP mice. $L$, CTB-Alexa594 (red) labeling of all RGC axons. GFP + axons were restricted to the ISGS as in wild-type CB2-GFP mice. $\boldsymbol{M}-\boldsymbol{R}$, Electron micrographs of P8 GFP + boutons located in the USGS at 5 h after enucleation. In the control SC hemisphere ( $\boldsymbol{M}-\mathbf{0}$ ), intact boutons with synapses (arrowhead, inset) were observed $(M, N) .0,3 D$ reconstruction of serial sections (17 serial sections including $M$ and $N ; 1.2 \mu \mathrm{m}$ thick) demonstrating the synaptic contact (green, bouton; purple, postsynaptic profile; yellow, PSD; gray square, section plane). In the enu. SC (P-R), GFP+ axon fragments were surrounded by glial processes (blue). $\boldsymbol{R}, 3 \mathrm{D}$ reconstructions (14 serial sections including $\boldsymbol{P}$ and $\mathbf{Q}$; (Figure legend continues.) 
A

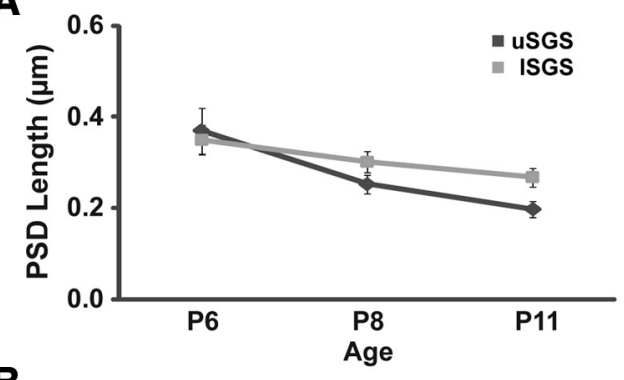

B

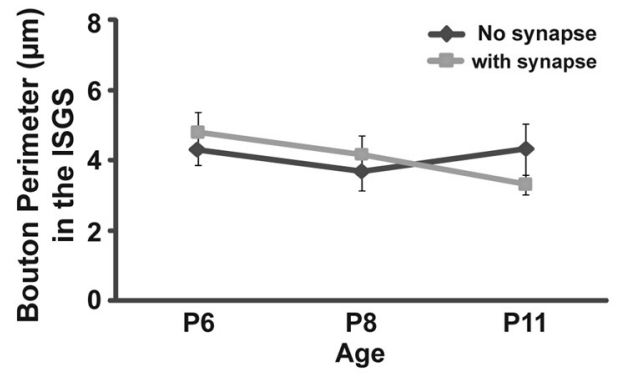

C

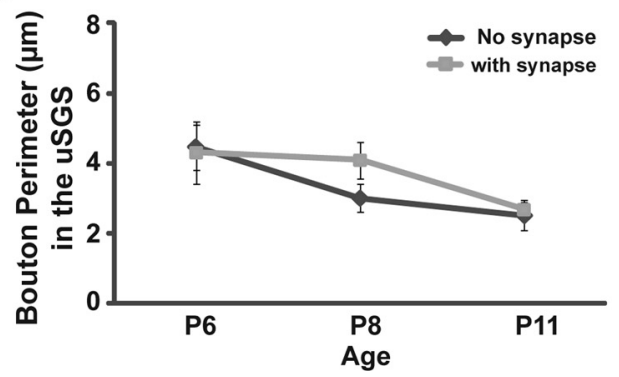

Figure 4. Quantification of OFF- $\alpha \mathrm{RGC}$ axonal boutons in the developing SC. A, PSD length as a function of age and laminar position. PSD length was not significantly different in the USGS versus ISGS ( $p=0.33$, two-way ANOVA; $n=5-9)$. $\boldsymbol{B}$, ISGS bouton perimeter as a function of age and synaptic contact. In the ISGS, the size of boutons stayed the same from P6 through P11 regardless of the presence or absence of synapses ( $p=0.72$, two-way ANOVA; $n=5-9)$. C, USGS bouton perimeter as a function of age and synaptic contact. In the USGS, bouton size decreased, regardless of the presence or absence of synapse (age $p=0.02$, synapse $p=0.36$, two-way ANOVA; $n=5-9$ ). Values are mean \pm SEM.

over time using time-lapse imaging, comparison of GFP + boutons from different-aged mice revealed that bouton size in the ISGS did not vary significantly as a function of synaptic contact (Fig. $4 B)(p=0.72$, two-way ANOVA, $n=5-9)$. In the uSGS, GFP + boutons steadily decreased in size from P6 through P11, regardless of whether they contained a synapse or not (Fig. 4C) (age $p=0.02$, synapse $p=0.36$, two-way ANOVA; $n=5-9$ ). These data indicate that synaptic parameters are not correlated with the stability of OFF- $\alpha$ RGC axonal arbors during laminaspecific refinement.

\section{Discussion}

The findings shown here provide several new insights into cellular events that give rise to lamina-specific axon connections in the mammalian CNS. Although our analysis was limited to OFF-

$\leftarrow$

(Figure legend continued.) $0.98 \mu \mathrm{m}$ thick) showing GFP + axon fragments (green) separated from mitochondria (pink), both of which are contained within the glial cell (blue). Scale bars: $\boldsymbol{A}-\boldsymbol{D}, \boldsymbol{K}, \boldsymbol{L}, 100 \mu \mathrm{m} ; \boldsymbol{E}-\mathbf{I}, 25 \mu \mathrm{m} ; \boldsymbol{M}-\mathbf{Q}, 0.5 \mu \mathrm{m}$; inset in $\boldsymbol{M}, 0.2 \mu \mathrm{m}$ (in main panels). Also see supplemental movie (available at www.jneurosci.org as supplemental material). $\alpha$ RGCs, we have reason to believe our findings apply to other RGC subtypes as well because both classic anatomical studies (Sachs et al., 1986) and genetic labeling approaches (Huberman et al., 2008a; Kim et al., 2010) (Fig. 1) show that various RGC subtypes establish lamina-specific connections by refining their axonal arbors. Here we show that OFF- $\alpha$ RGC axons achieve lamina-specific refinement without undergoing degeneration (Fig. 3). It is unlikely that we failed to observe degeneration or glial engulfment due to technical limitations, because after we axotomized one optic nerve, we could readily observe degeneration and GFP + axonal debris inside glial cells (Fig. 3). The absence of axonal degeneration under normal developmental conditions, combined with the fact that lamina-specific targeting of OFF- $\alpha$ RGCs ensues after naturally occurring RGC death (Farah and Easter, 2005), argues that lamina-specific OFF- $\alpha$ RGC refinement is achieved in large part by retraction of axon arbors that initially project to both appropriate and inappropriate target laminae.

Another novel finding shown here is that lamina-specific axon targeting involves the coincident formation of synapses within both correct and incorrect target laminae (Fig. 2). This is reminiscent of the binocular innervation of target neurons that occurs during early stages of eye-specific refinement (Campbell and Shatz, 1992; Jaubert-Miazza et al., 2005). Despite this similarity, however, eye-specific refinement and laminar-specific refinement of functionally distinct RGC subtypes are mechanistically distinct in certain ways. In particular, whereas manipulating spontaneous RGC activity or the activity of target neurons has deleterious effects on eye-specific refinement (Huberman et al., 2008b), many of those same manipulations (e.g., pharmacologically or genetically disrupting cholinergic transmission) fail to prevent lamina-specific refinement of axons from distinct RGC subtypes (Huberman et al., 2008a). Indeed, in chickens and zebrafish, lamina-specific targeting of retino-SC axons appears independent of synaptic transmission altogether (Inoue and Sanes, 1997; Nevin et al., 2008). Thus, lamina-specific refinement of axons arising from functionally distinct sources reflects a distinct process that includes the formation and elimination of synaptic contacts but that does not appear to rely on synaptic transmission per se.

A related and somewhat surprising aspect of our findings is that the presence and maintenance of a structural synapse does not correlate with the stability of a given axonal arbor (Fig. 4). This observation, combined with the apparent activityindependence of lamina-specific targeting, suggests that arbor stability during lamina-specific refinement is not regulated by synaptic transmission. Molecular cues present at synaptic junctions but that do not rely on synaptic transmission may be essential to form and maintain lamina-specific synaptic connections. Adhesive and repellant cues have been implicated in generating synaptic specificity in other systems (Chen and Cheng, 2009; Huberman et al., 2010), and some of them, such as the cadherins, show synaptic localization (Takeichi, 2007). Thus, in future studies it will be interesting to explore the localization of adhesion molecules during lamina-specific targeting and determine whether their expression is modulated by forms of activity such as calcium waves (Torborg and Feller, 2005). In the meantime, the data shown here provide a framework for understanding the type and scale of cell-cell interactions that generate lamina-specific synaptic connectivity of functionally distinct cell types in the mammalian CNS. 


\section{References}

Bickford ME, Slusarczyk A, Dilger EK, Krahe TE, Kucuk C, Guido W (2010) Synaptic development of the mouse dorsal lateral geniculate nucleus. J Comp Neurol 518:622-635.

Botto M, Dell'Agnola C, Bygrave AE, Thompson EM, Cook HT, Petry F, Loos M, Pandolfi PP, Walport MJ (1998) Homozygous C1q deficiency causes glomerulonephritis associated with multiple apoptotic bodies. Nat Genet 19:56-59.

Campbell G, Shatz CJ (1992) Synapses formed by identified retinogeniculate axons during the segregation of eye input. J Neurosci 12:1847-1858.

Chen SY, Cheng HJ (2009) Functions of axon guidance molecules in synapse formation. Curr Opin Neurobiol 19:471-478.

Clandinin TR, Zipursky SL (2002) Making connections in the fly visual system. Neuron 35:827-841.

Edwards MA, Caviness VS Jr, Schneider GE (1986) Development of cell and fiber lamination in the mouse superior colliculus. J Comp Neurol 248:395-409.

Farah MH, Easter SS Jr (2005) Cell birth and death in the mouse retinal ganglion cell layer. J Comp Neurol 489:120-134.

Faulkner RL, Jang MH, Liu XB, Duan X, Sailor KA, Kim JY, Ge S, Jones EG, Ming GL, Song H, Cheng HJ (2008) Development of hippocampal mossy fiber synaptic outputs by new neurons in the adult brain. Proc Natl Acad Sci U S A 105:14157-14162.

Huberman AD, Manu M, Koch SM, Susman MW, Lutz AB, Ullian EM, Baccus SA, Barres BA (2008a) Architecture and activity-mediated refinement of axonal projections from a mosaic of genetically identified retinal ganglion cells. Neuron 59:425-438.

Huberman AD, Feller MB, Chapman B (2008b) Mechanisms underlying development of visual maps and receptive fields. Annu Rev Neurosci 31:479-509.

Huberman AD, Wei W, Elstrott J, Stafford BK, Feller MB, Barres BA (2009) Genetic identification of an On-Off direction selective retinal ganglion cell subtype reveals a layer-specific subcortical map of posterior motion. Neuron 62:327-334.

Huberman AD, Clandinin TR, Baier H (2010) Molecular and cellular mechanisms of lamina-specific axon targeting. Cold Spring Harb Perspect Biol 2:a001743.
Inoue A, Sanes JR (1997) Lamina-specific connectivity in the brain: regulation by N-cadherin, neurotrophins, and glycoconjugates. Science 276:1428-1431.

Jaubert-Miazza L, Green E, Lo FS, Bui K, Mills J, Guido W (2005) Structural and functional composition of the developing retinogeniculate pathway in the mouse. Vis Neurosci 22:661-676.

Kim IJ, Zhang Y, Meister M, Sanes JR (2010) Laminar restriction of retina ganglion cell dendrites and axons: subtype-specific developmental patterns revealed with transgenic markers. J Neurosci 30:1452-1462.

McLaughlin T, O’Leary DD (2005) Molecular gradients and development of retinotopic maps. Annu Rev Neurosci 28:327-355.

Meissirel C, Wikler KC, Chalupa LM, Rakic P (1997) Early divergence of magnocellular and parvocellular functional subsystems in the embryonic primate visual system. Proc Natl Acad Sci U S A 94:5900-5905.

Nevin LM, Taylor MR, Baier H (2008) Hardwiring of fine synaptic layers in the zebrafish visual pathway. Neural Dev 3:36.

Sachs GM, Jacobson M, Caviness VS Jr (1986) Postnatal changes in arborization patterns of murine retinocollicular axons. J Comp Neurol 246:395-408

Sanes JR, Yamagata M (2009) Many paths to synaptic specificity. Annu Rev Cell Dev Biol 25:161-195.

Siegert S, Scherf BG, Del Punta K, Didkovsky N, Heintz N, Roska B (2009) Genetic address book for retinal cell types. Nat Neurosci 12:1197-1204.

Stevens B, Allen NJ, Vazquez LE, Howell GR, Christopherson KS, Nouri N, Micheva KD, Mehalow AK, Huberman AD, Stafford B, Sher A, Litke AM, Lambris JD, Smith SJ, John SW, Barres BA (2007) The classical complement cascade mediates CNS synapse elimination. Cell 131:1164-1178.

Takeichi M (2007) The cadherin superfamily in neuronal connections and interactions. Nat Rev Neurosci 8:11-20.

Tessier-Lavigne M, Goodman CS (1996) The molecular biology of axon guidance. Science 274:1123-1133.

Torborg CL, Feller MB (2005) Spontaneous patterned activity and the refinement of retinal projections. Prog Neurobiol 76:213-235.

Yonehara K, Ishikane H, Sakuta H, Shintani T, Nakamura-Yonehara K, Kamiji NL, Usui S, Noda M (2009) Identification of retinal ganglion cells and their projections involved in central transmission of information about upward and downward image motion. PLoS One 4:e4320. 